\title{
Categorización de los énfasis de los proyectos de investigación en malaria financiados por Colciencias durante 1995-2005
}

\author{
Classification of the scope of research projects on malaria funded \\ by Colciencias during 1995-2005
}

Beatriz Macías-Angel, Carlos A. Agudelo C y María M. Ronderos-Torres

Recibido 10 julio 2016/Enviado para Modificación 3 septiembre 2016/Aceptado 14 noviembre 2016

\section{RESUMEN}

BM.: Microb. M.Sc. Microbiología. Ph.D. Doctorado Interfacultades Salud Pública. Universidad Nacional de Colombia. Bogotá. Escuela de Medicina y Ciencias de la Salud, Universidad del Rosario. Bogotá, Colombia.bpmaciasa@unal.edu.co CA.: MD. M.Sc. Salud Pública. M.Sc. Ciencias. Instituto de Salud Pública, Facultad de Medicina, Universidad Nacional de Colombia.caagudeloc@unal.edu.co

MR.: MD. M.Sc. Epidemiología. Pontificia Universidad Javeriana, Bogotá. Colombia. ixtchala@hotmail.com

Objetivo Identificar los principales objetivos y énfasis de los proyectos de investigación en malaria financiada por Colciencias entre 1995 y 2005.

Métodos Para la identificación del énfasis de los proyectos de investigación en malaria se utilizó el sistema de clasificación en investigación en salud desarrollado por la Colaboración de Investigación Clínica de Gran Bretaña: "Health Research Classification System" (HRCS).

Resultados Se encontró que la investigación en malaria durante este periodo tiene énfasis en determinantes y causas, las principales ciudades beneficiadas con la financiación de proyectos de investigación en malaria son instituciones pertenecientes a Bogotá, Medellín y Cali.

Conclusiones Estos proyectos permitieron el fortalecimiento en núcleos de investigación en malaria en las líneas de biología molecular, producción de vacunas, identificación de resistencia a antimaláricos y estudios relacionados con el cambio climático; algunos de sus resultados con capacidad de afectar políticas en malaria y formación de recurso humano.

Palabras Clave: Evaluación del impacto en salud, proyectos de investigación e innovación, evaluación (fuente: DeCS, BIREME).

\section{ABSTRACT}

Objective To identify the main focus of research projects on malaria funded by Colciencias between 1995 and 2005.

Methodology The Health Research Classification System (HRCS), developed by The UK Clinical Research Collaboration (UKCRC), was used for identifying the emphasis of research works on Malaria.

Results During this period, research on malaria was mainly directed to etiology; the main cities benefited by the funding of research projects on malaria are institutions from Bogotá, Medellín and Cali.

Conclusions These type of projects improve the strengthening of malaria research centers in areas such as molecular biology, production of vaccines, identification of resistance to antimalarial treatment, and climate change; some of the results obtained can affect policies and training of human resources on malaria.

Key Words: Health impact assessment, research and innovation projects, evaluation (source: MeSH, NLM).

DOI: https://doi.org/10.15446/rsap.v19n1.58957 
$\mathrm{E}$ n el contexto mundial la malaria es un problema grave de salud pública, que causa la muerte aproximadamente a I.I millones de personas por año según la Organización Mundial de la Salud-OMS (I). En Colombia, más de 25 millones de personas habitan en zonas endémicas (2), esta enfermedad presenta manifestaciones crónicas y agudas en el hombre, producidas por especies Plasmodium falcipaparum, $P$. vivax, $P$. malariae y $P$. ovale.

En los últimos 40 años la malaria ha sido uno de los problemas prioritarios en salud y es por esta razón que Colciencias siendo el Departamento Administrativo de Ciencia, Tecnología e Innovación en Colombia en el trascurso de las dos pasadas décadas ha invertido recursos en proyectos de investigación en malaria, pues la investigación en salud se ha constituido como uno de los pilares de progreso y bienestar de nuestra sociedad.

Colciencias es creado en I968 bajo la influencia internacional de lo que para entonces se conociera como "el movimiento internacional para la aplicación de la ciencia y la tecnología a los problemas del desarrollo" conformado principalmente por organismos internacionales como las Naciones Unidas, UNESCO, OEA y IDRC (3). Desde I 968 al 2008 se han identificado seis periodos en Colciencias relacionados con la política de Ciencia y Tecnología e investigación en salud con el fin de producir y aportar cambios en la toma de decisiones que generen impactos en la salud, la economía y el bienestar social (3).

Esta entidad realiza grandes esfuerzos financiando proyectos de malaria desde distintos enfoques como el básico-clínico hacia I995; el biomédico que predomino entre I995 y el 2000; estudios principalmente clínicos y epidemiológicos hacia el 2007 (3).

La malaria causa el I,3\% de pérdida del crecimiento económico anual en los países donde presenta una transmisión intensa. La evidencia acumulada muestra que la malaria no es consecuencia de la pobreza, sino una causa de pobreza persistente; en este entorno se puede decir que Colombia sigue inmerso en las desigualdades sociales y culturales que hacen de esta sociedad una comunidad vulnerable al flagelo de la malaria $(4,5)$.

Es necesario conocer a profundidad los objetivos de los pasados proyectos con el fin de identificar fortalezas y vacíos de la investigación en malaria en Colombia que orienten la inversión futura de Colciencias.

El sistema de clasificación en investigación en salud "Health Research Classification System (HRCS)" es un sistema para clasificar y el analizar la financiación de la investigación biomédica y en salud, desarrollado por "The UK Clinical Research Collaboration (UKCRC") (8) y fue implementado en el 2005 por organismos de financiación biomédica del Reino Unido con el fin de identificar los temas de investigación en cáncer; en el 20 Io se utiliza nuevamente con el propósito de identificar las tendencias de investigación a través del tiempo (9).

El HRCS se ha utilizado para conocer el panorama de los proyectos financiados, analizar las brechas del conocimiento e identificar las oportunidades de investigación en Bogotá y en países Europeos (Io). Con este trabajo se pretende aportar al conocimiento de la actividad investigativa realizada en Colombia con financiación de Colciencias sobre el problema de la malaria en el decenio I995-2005; de esta manera se espera brindar información de utilidad para la toma de decisiones en la utilización futura de recursos públicos destinados a entender el comportamiento de la malaria, sus determinantes y las intervenciones efectivas para su control.

\section{MATERIALES Y MÉTODOS}

Para la clasificación de los proyectos de investigación en malaria financiados por Colciencias se utilizó el Sistema de Clasificación en Investigación en Salud "Health Research Classification System" (HRCS) (8).

El Centro de Documentación de Colciencias suministró una base de datos sobre los proyectos financiados durante I995 y 2005; en esta se identificaron 584 proyectos financiados para el área de la salud por recuperación contingente que es la línea de financiamiento que se otorga a proyectos cuyo desarrollo no genera beneficios económicos directos. En el estudio se incluyeron 20 proyectos financiados por Colciencias con o sin contrapartida, que respondían a un problema de investigación relacionado con malaria a nivel nacional, regional o local y que contaron con el informe final.

Los objetivos de los proyectos son incluidos en una hoja de cálculo para luego ser clasificados y analizados por dos expertos codificadores quienes para asegurar el resultado realizaron un segundo pase de codificación que permite confirmar los datos finales, cada proyecto se clasifico con un máximo de dos códigos de actividad de investigación y los montos de financiación se ajustaron a precios constantes del 2005para ser analizados.

El sistema de Clasificación aborda dos dimensiones, la primera, permite identificar la actividad de investigación y la otra clasifica la investigación de acuerdo con el área de la salud (8).

Los códigos describen 8 actividades de investigación así: El código I.o hace referencia a fundamentación, el 2.o Etiología, el 3.o Prevención de enfermedades, condiciones y promoción del bienestar, el 4.o Detección, evaluación y diagnóstico, el 5.o Desarrollo de tratamientos e intervenciones terapéuticas, el 6.o Evaluación de trata- 
mientos e intervenciones terapéuticas, el 7.0 Manejo de la enfermedad y sus condiciones y el 8.o Políticas, sistemas, servicios sociales y de salud (8).

Las categorías principales se subdivide en 48 sub-códigos de actividades de investigación, en el presente estudio se analizan los códigos principales de las categorías y los sub-códigos con el fin de conocer las principales actividades de investigación (8).

\section{RESULTADOS}

Entre 1995 y el 2005 Colciencias financió por recuperación contingente cerca 547 proyectos en salud, 35 de los cuales se asociaron con objetivos de malaria liderados por 26 investigadores y ejecutados por 9 entidades públicas y privadas de Bogotá, Bucaramanga, Cali, Cartagena, Medellín y Sabaneta, se consultaron 20 informes finales de investigación.

Las ciudades más beneficiadas con la financiación de Colciencias entre 1995 y 2005 fueron Medellín y Cali con Io y 7 proyectos cada una y con una participación del $44 \%$ y $33 \%$, Bogotá con un 21 \% en un solo proyecto siendo este el proyecto de mayor financiación en malaria de Colombia y 0,44 \% en un segundo; Sabaneta recibe una mínima financiación $(0,44 \%)$ del total invertido en los 20 proyectos de malaria durante este periodo (\$4.198.896.05 de pesos a precios constantes del 2005).

No incluimos los datos de 3 proyectos financiados por Colciencias en 1995 debido a que no se pudieron consultar los informes finales. Los años que mayor financiación recibieron para proyectos de malaria son 1996 (\$889.683.6I8 de pesos a precios constantes del 2005) y 2004 (\$ I.267.683.709 de pesos a precios constantes del 2005) y los que menos subvención recibieron son I 999 (\$55.46I.I70 de pesos a precios constantes del 2005) y 2000 (67659.023 de pesos a precios constantes del 2005).
Se identificaron 5 categorías y Io subcategorías de actividades de investigación en los 20 proyectos de malaria financiados por Colciencias durante este periodo (Tabla I).

En la actividad de investigación sobre productos farmacéuticos se clasificaron 7 proyectos con el código 6.I que hace referencia a investigaciones que están relacionadas con la aplicación clínica, evaluación de pequeñas moléculas farmacéuticas, vacunas, anticuerpos y hormonas en los seres humanos, incluyendo ajustes en pequeña escala, estudios piloto y ensayos de fase I, II, III y IV. Se encontró que en este periodo se han realizado estudios de evaluación sobre la capacidad de medicamentos antimaláricos, la proporción de fallas terapéuticas para algunos medicamentos, la presencia de alelos, genes de resistencia y sensibilidad en P.facilparum y P.vivax y se realiza una investigación en una población indígena de Turbo. La categoría de etiología del código 2.0 conto con 7 proyectos que corresponde a los estudios que identifican los factores determinantes que intervienen en la causa, riesgo o desarrollo de la enfermedad, las condiciones y los problemas de salud; estos se subclasificaron en actividades de investigación relacionadas con el código 2.I factores biológicos y endógenos, 2.2 factores relacionados con el medio ambiente, 2.3 factores psicológicos, sociales y económicos y el código 2.4 vigilancia y distribución (estudios de observación, encuestas, registros y estudios que rastrean la incidencia, prevalencia, morbilidad, comorbilidad y mortalidad incluyendo el monitoreo continuo). Estas siete propuestas de investigación analizan varios de los componentes etiológicos de la malaria convirtiéndose en un enfoque predominante de la investigación financiada por Colciencias, como se ilustra en las Figuras i y 2.

En la actividad de investigación relacionada con la prevención de enfermedades, condiciones y promoción del bienestar con dos subcategorías se identificaron 3 proyectos.

Tabla 1. Descripción código de las actividades y número de proyectos financiados

\begin{tabular}{|c|c|c|}
\hline Código & Actividad & $\begin{array}{c}\text { No de } \\
\text { proyectos }\end{array}$ \\
\hline 1.0 & $\begin{array}{l}\text { Fundamentación: son aquellas investigaciones que permiten sustentar la causa, el desarrollo, la detección, el tratamiento y } \\
\text { control de enfermedades, condiciones y problemas de salud. }\end{array}$ & 0 \\
\hline 2.0 & $\begin{array}{l}\text { Etiología: son aquellos estudios que incluyen la identificación de los factores determinantes que intervienen en la causa, } \\
\text { riesgo o desarrollo de la enfermedad, las condiciones y los problemas de salud. }\end{array}$ & 7 \\
\hline 3.0 & $\begin{array}{l}\text { Prevención de enfermedades, condiciones y promoción del bienestar: la investigación está encaminada a la prevención } \\
\text { primaria de enfermedades, dolencias, enfermedades profesionales y a la promoción del bienestar. }\end{array}$ & 3 \\
\hline 4.0 & $\begin{array}{l}\text { Detección, Evaluación y Diagnóstico: investigaciones relacionadas con proyectos que determinan el desarrollo y evalúan los } \\
\text { pronósticos de diagnóstico, los marcadores predictivos y las tecnologías. }\end{array}$ & 1 \\
\hline 5.0 & $\begin{array}{l}\text { Desarrollo de tratamientos e intervenciones terapéuticas: descubrimiento y desarrollo de intervenciones terapéuticas, pruebas } \\
\text { en modelos, sistemas y entornos preclínicos. }\end{array}$ & 1 \\
\hline 6.0 & $\begin{array}{l}\text { Evaluación de tratamientos e intervenciones terapéuticas: pruebas y evaluación de intervenciones terapéuticas en clínica, en } \\
\text { comunidad y en ensayos. }\end{array}$ & 8 \\
\hline 7.0 & $\begin{array}{l}\text { Manejo de la enfermedad y sus condiciones: investigaciones sobre las necesidades de atención, manejo de la enfermedad } \\
\text { sus condiciones y los problemas de salud. }\end{array}$ & 0 \\
\hline 8.0 & $\begin{array}{l}\text { Políticas, sistemas, servicios sociales y de salud: investigaciones sobre el suministro, la asistencia sanitaria y social de } \\
\text { servicios incluyendo políticas de salud, estudios de diseño de investigación, medidas y metodologías. }\end{array}$ & 0 \\
\hline
\end{tabular}


Figura 1. Distribución del financiamiento de los proyectos de malaria por actividades de Investigación, financiados por Colciencias entre 1995 y 2005

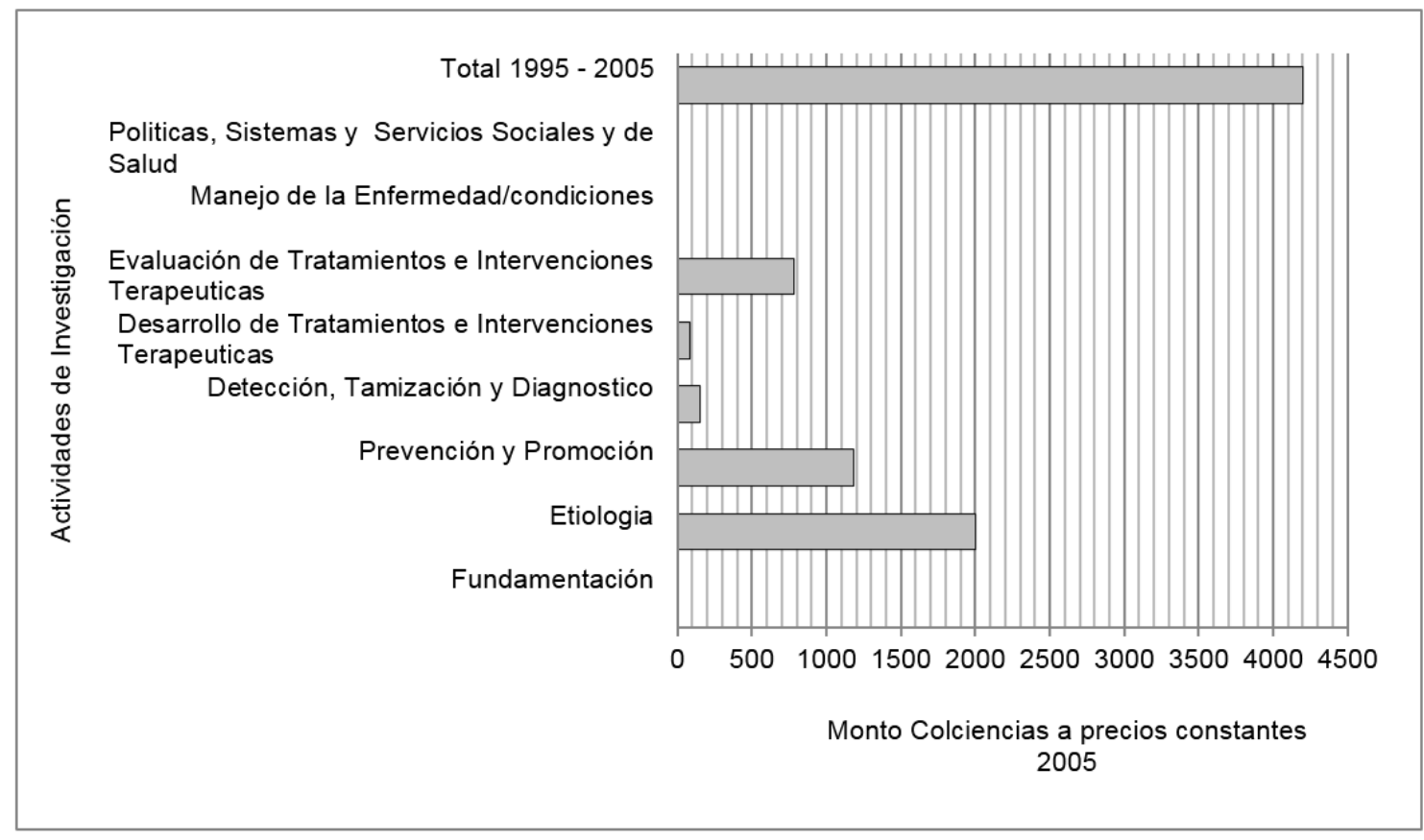

Con el código 3.2 sobre intervenciones para modificar los riesgos ambientales físicos y biológicos se relacionó solo un proyecto; 2 se asociaron con el código 3.4 en vacunas relacionado con la investigación sobre las vacunas para la prevención de la malaria.

En la actividad de investigación con el código 4.0 sobre detección, evaluación y diagnóstico que hace referencia a investigaciones relacionadas con proyectos que determinan el desarrollo y evalúan los pronósticos de diagnóstico, los marcadores predictivos y las tecnologías solo se identificó un proyecto en la subcategoría que hace referencia a tamizajes en la población.

La actividad del código 5.0 que se refiere al desarrollo de tratamientos e intervenciones terapéuticas se clasifico un proyecto en la subcategoría el código 5. I sobre desarrollo de productos farmacéuticos.

En cuanto a la distribución de financiamiento a través de actividades de investigación esta es diferente y no se asocia al número de proyectos financiados por actividad sino al monto financiado por actividad como lo observamos en la Figura 2.

Figura 2. Distribución de los recursos otorgados por Colciencias a proyectos de investigación en malaria según actividad de investigación del HRCS 1995-2005

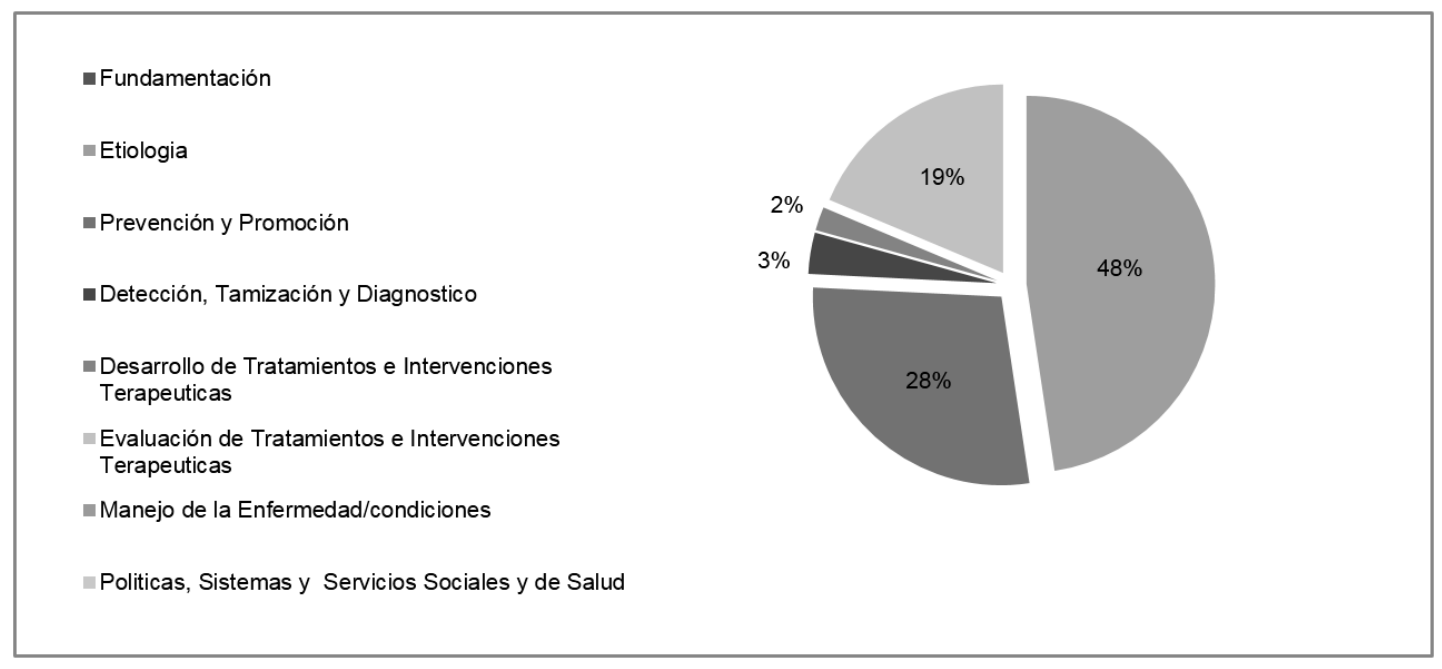


Prevención y promoción se constituyen en la segunda actividad de investigación financiada por Colciencias, evaluación de tratamientos e intervenciones terapéuticas ocupa un tercer lugar de relevancia. Durante este período no se identificaron proyectos de investigación en malaria que tuviesen objetivos dirigidos a "Fundamentación (código I)", "Manejo de la enfermedad (código 7)" y "políticas, sistemas y servicios sociales (código 8)".

Las actividades de investigación durante I995-2000 y del 200I-2005 guardan similitud en cuanto a los aportes de investigación siendo mayor la financiación del 200I al 2005.

Podemos decir que del total de financiamiento otorgado por Colciencias para los 20 proyectos de malaria estudiados en este período, los recursos se distribuyeron según la actividad investigativa de la siguiente manera: 48 \% (\$ I 999 427055 de pesos a precios constantes del 2005) en etiología; el $28 \%$ (\$ I I 8243 I 876 de pesos a precios constantes del 2005) a estudios de prevención y promoción; I9 \% (\$ 783635772 de pesos a precios constantes del 2005) a evaluación de tratamientos e intervenciones terapéuticas y una menor proporción a desarrollo de tratamientos e intervenciones terapéuticas (Figura 2). Cabe resaltar que en este periodo no se dio financiación a ningún proyecto dirigido a entender, evaluar o innovar en la organización y entrega de servicios de salud, políticas, aspectos económicos o servicios sociales en la problemática de malaria en el país.

\section{DISCUSIÓN}

Entre I995 y 2005 Colciencias privilegió en su distribución de recursos para la investigación en malaria los proyectos dirigidos a entender los factores y determinantes de la incidencia y prevalencia de malaria, siendo los estudios ambientales los que recibieron mayores aportes; en segundo lugar, los de factores biológicos y estudios de vigilancias y, en último lugar, encontramos un proyecto relacionado con los factores sociales y económicos.

Este $48 \%$ de los proyectos financiados permiten que la investigación en malaria se fortalezca, capacite constituya redes y líderes de investigación durante los siguientes 20 años en el área de Básicas y Ciencias Biomédicas; es aquí donde se presenta la mayor producción de conocimiento en cuanto a número de publicaciones, formación de estudiantes de Maestría y de Doctorado representados en Tesis que se constituyen en productos de gran impacto para las instituciones académicas que se fortalecen en sus proceso de acreditación, uno de los indicadores claves para su reconocimiento nacional e internacional (I I).

Cuando se analiza el proceso de financiación desde I995 a 2005 se evidencia la crisis en la financiación de proyectos durante los años I999 y 2000 en donde a pesar de financiarse 4 proyectos tres de ellos no superan los 20 millones de pesos cada uno, no se asocian con productos, resultados relacionadas en publicaciones o en otro nivel de impacto, aunque hacen parte de líneas de investigación. A partir del 200I se observa un aumento en la financiación de los proyectos relacionados con el Fondo de investigaciones en Salud (FIS) (I2).

Siete de los proyectos financiados se relacionan con estudios de resistencia a antimaláricos y estos en su conjuntos afectan de forma directa guías y políticas en malaria, como se evidencia en varias publicaciones en las que son gestores de nuevos protocolos de tratamiento, de georreferenciación de vectores de la malaria que fortalecen el conocimiento y los planteamientos de política del Ministerio de Salud en cuanto a manejo y tratamiento de la malaria. De esto hacen parte entidades relacionadas con los proyectos 3, 6, y I 5 (I3, I4).

Existe una participación importante en la estandarización de moléculas con el propósito de producir vacunas para la malaria. Esto ha dejado como resultado la producción de moléculas con buenas prácticas de manufactura, la estandarización de procesos para prueba en pacientes con estándares internacionales; sin embargo, ninguna de las relacionadas en los proyectos ha sido patentada y como se indica en varias publicaciones son pocas las moléculas patentadas con estas características de vacuna en Colombia. Los que lideran las patentes en salud en Colombia se encuentran en las áreas del manejo de señales, de filtros implantables en los vasos sanguíneos, prótesis, ortopédicas, cuidado o dispositivos anticonceptivos, tratamiento o protección de ojos o de oídos, vendajes, preparaciones o cojines absorbentes y kits de primeros auxilios, en los últimos 20 años (I5) •

Agradecimientos: Los autores expresan su agradecimiento al Programa Interfacultades Doctorado en Salud Pública y al Instituto de Salud Pública de la Universidad Nacional de Colombia; al Programa Nacional de Salud de Colciencias por su apoyo y colaboración para la ejecución de este proyecto.

\section{REFERENCIAS}

1. WHO (World Health Organization). World Health Report. Geneva: WHO; 2002.

2. Valero-Bernal MV. Malaria in Colombia: Retrospective Glance during the Past 40 Years. Rev. Salud Pública. 2006; 8 (3):141-149.

3. Jaramillo H, Lopera C, González BE, Vecino A. Impacto del financiamiento en investigación en salud Colciencias 1970 2007. Informe final. Bogotá, Colciencias; agosto 2009.

4. Breman J. The ears of the hippopotamus: manifestations, determinants, and estimates of the malaria burden. American Journal of Tropical Medicine and Hygiene. 2001; 64(12): 1-11. 
5. Granados-Torano R. Reformas Neolibreales de los Sistemas de Salud. Facultad Nacional de Salud Pública Carlos Finlay. La Habana: Instituto Superior de Ciencias Médicas de La Habana: 2001 p. 3000

6. Organización de las Naciones Unidas. Objetivos de Desarrollo del Milenio Informe de 2013. Nueva York. USA

7. Instituto Nacional de Salud. Protocolo de vigilancia en salud pública de la malaria. Disponible en: http://www.ins.gov.co/?idcategoria=49752\#. Consultado en febrero de 2014

8. UK Clinical Research Collaboration. Health Research Classification System (HRCS). London, UK: UKCRC; 2011

9. Terry et al. Mapping global health research investments, time for new thinking - A Babel Fish for research data. Health Research Policy and Systems. 2012;10:28.

10. Research Council of Norway Health Research Classification System (HRCS): Harmonization and Further Development of the Use in Norway. Oslo: Research Council of Norway; 2014.
11. Mineducacion.gov.co. Por primera vez Gobierno MIDE la calidad de la educación superior en el país - Centro Virtual de Noticias de Educación [Internet]. 2015 [cited 27 November 2015].

12. Trillos-Peña CE, Latorre-Santos $C$, Ortega JA. Perspectiva de la investigación en la Secretaria Distrital de Salud. Investig. segur. soc. salud, 2006; 8(8):9-33.

13. Guía 19. Guías de promoción de la salud y prevención de enfermedades en la salud pública. Programa de Apoyo a la Reforma de Salud/PARS. Ministerio de la Protección Social; 2005.

14. Segura O. Terapia farmacológica antipalúdica en Colombia recomendaciones de consenso. Reunión sobre política de antimalaricos en Colombia 28-30 de septiembre de Ministerio de la Protección Social. República de Colombia. Dirección General de Salud Pública. Grupo de Promoción y Prevención; 2004.

15. Sánchez J, Medina J, León A. Publicación internacional de patentes por organizaciones inventores de origen colombiano. Cuadernos de Economía., 26 (47), 247-270, 2007. 